\title{
Microstructural Characterization of WC-Co-hBN Cemented Carbide Processed Using Selective Laser Sintering
}

\author{
Joseph Agyapong ${ }^{1}$, Aleksander Czekanski ${ }^{2}$ and Solomon Boakye-Yiadom ${ }^{2}$ \\ ${ }^{1}$ York University, Toronto, Ontario, Canada, ${ }^{2}$ York University, United States
}

Cemented carbide materials such as Tungsten Carbide alloy (WC-Co) are desired by many industrial companies including manufacturing and nuclear industries for their parts due to the material's ability to retain mechanical properties (strength) at high temperatures [1]. However, due to its high melting point, WC-Co parts are made using conventional powder metallurgy (PM) methods. These methods are known to require a high level of skill and simplicity of the part about to fabricated. Additionally, obtaining a WC-Co good part made using PM requires a huge amount of time which also becomes an arduous task to complete [2]. This has made it a little difficult in adopting innovation and intricacy of designs for WC-Co parts which may meet today's industrial problems. Additionally, WC-Co has long been thought of as a material for consumer goods [2] such as belt hooks, rings, knives among others but the inconvenient processing route makes it difficult to adopt it for these consumer goods. In a bid to improve the processing route, Powder Bed Fusion (PBF) techniques such as Selective laser melting (SLM) and Selective laser sintering (SLS) have been considered [3]. The benefit of lower production time, the flexibility and versatility of components designed and created with lower densities, make it even more desirable [3]. The attempt to print WC-Co using 3D printing was groundbreaking even though most researchers discovered more complex quandaries with the process [3]. Problems such as rough surface finish, residual stresses, non-equilibrium phases and micro cracks come up due to uncontrolled, repeated and irregular thermal cycles induced during processing. In a bid to improve the wear properties and non-equilibrium phases, researchers have looked at tailoring the microstructure of the material by optimizing the printing parameters during processing and adopting a one-step post-processing treatment after printing [4,5]. However, the improved wear resistance values of their 3D printed samples were weaker than the conventionally manufactured ones $[4,5]$. In this study, we wish to improve the mechanical properties (wear resistance) and avoid non-equilibrium phases of the 3D printed WC-Co by adding a reinforcement which is precisely hexagonal Boron Nitride $(\mathrm{hBN})$, a $2 \mathrm{D}$ material known as a high temperature performing material. Additionally, it is more attractive as an additive material for machining tools because of its self-lubricating properties [6]. An initial study was done to select the appropriate powders and select the optimum printing parameters suitable for printing a consolidated WC-Co-hBN part. After, printing, extensive microstructural characterization techniques including electron microscopy and a tribological experiment was conducted to ascertain the structure and properties of the printed material.

From the microscopy results (Fig 1), the as-printed WC-Co-hBN part exhibited a complex microstructure as a result of the processing conditions. Generally, 3 distinct structures including carbide chips, pure metallic binder regions and an eta phase. There was a large variation in the sizes of carbide chips ranging from 8um to 18um and identified using Energy Dispersive Spectroscopy (EDS) as WC carbides. These sizes are smaller relative to an SLS printed WC-17Co [7]. The eta phase regions were devoid of micropores unlike the WC carbides. Xray diffraction showed $\mathrm{WC}$ as a major phase and minor phases such as W3Co3C, W3Co3N, W9Co3C4, CoWB, Co5.47N phases (Fig. 2). This variety in phases comes up due to the processing condition which generated repeated thermal cycles and high cooling rates. However, no unstable W2C phase was identified in the system which demonstrates that h-BN addition inhibits $\mathrm{W} 2 \mathrm{C}$ formation. Tribological experiments showed better wear resistance for this material than the as-printed WC-Co and conventionally manufactured WC-Co attributed to the self-lubricating properties of h-BN. 


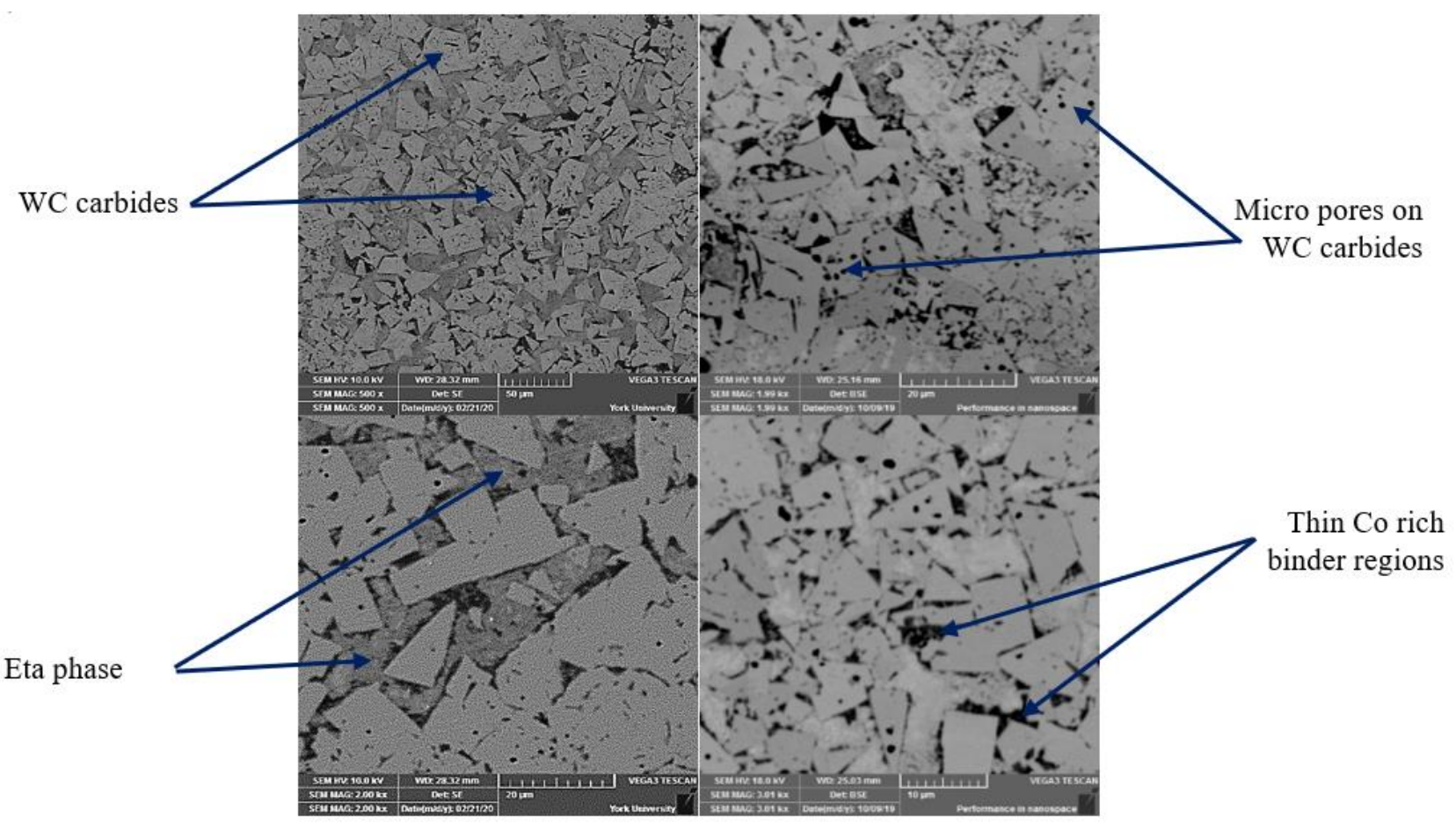

Figure 1. Figure 1: Scanning Electron micrographs for as-printed sample describing the microstructural features and discontinuities identified as a result of processing technique.

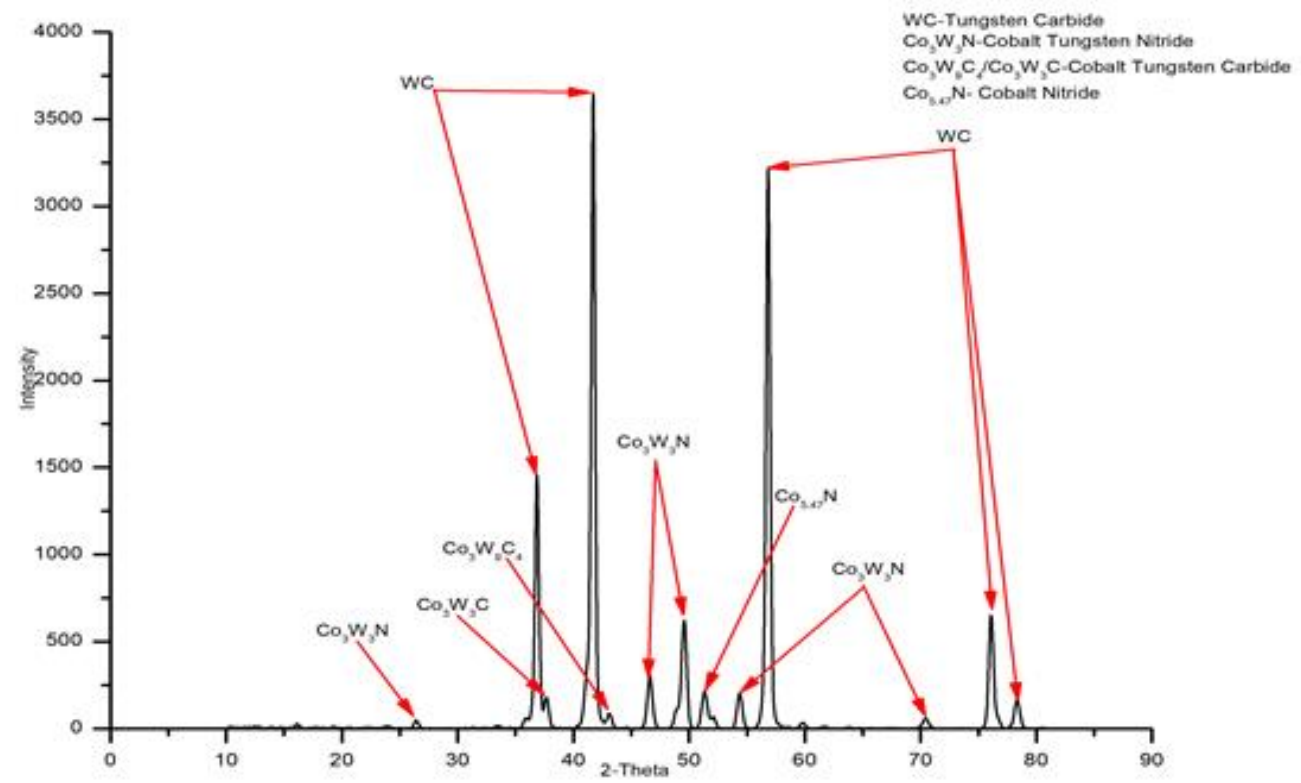

Figure 2. Figure 2: XRD pattern for as-printed sample describing the phases and their volume fraction as a result of processing technique.

\section{References}

[1] Fang, Zak Z., Xu W., Ryu T., Hwang K. S., Sohn H., "Synthesis, Sintering, and Mechanical, Properties of Nanocrystalline Cemented Tungsten Carbide - A Review.” I.J.R.M., (2009) vol 2, pp.: 288-99. 
[2] Colin, C, L Durant, N Favrot, J Besson, G Barbier, and F Delannay, "Processing of Functional-Gradient WC-Co Cermets by Powder Metallurgy." I.J.R.M. (1993), vol 12 pp. 145-52

[3] Uhlmann, E., Bergmann, A Gridin, Procedia CIRP (2015), Vol. 35, pp. 8-15.

[4] Jian C., Huang M., Fang Z., Koopman M., Liu W., et al, "Microstructure Analysis of High-Density WCCo Composite Prepared by One Step Selective Laser Melting." I.J.R.M. (2019), Vol. 84

[5] Campanelli, S. Luisa, N. Contuzzi, P. Posa, A. Angelastro, "Printability and Microstructure of Selective Laser Melting of WC/Co/Cr Powder.”, Materials,(2019) Vol: 12 pp. 2397

[6] Khatavkar, A. K. Mandave, D. D. Baviskar, L. Samir, "Influence of Hexagonal Boron Nitride on Tribological Properties of AA2024-HBN Metal Matrix Composite,” (2018), pp. 3792-98.

[7] Agyapong, J., Czekanski, A., Boakye-Yiadom S., Microstructural Characterization of WC-Co Cemented Carbide Processed Using Selective Laser Sintering. Microscopy and Microanalysis, 25(S2), (2019)2604-2605 